\title{
Autologous dendritic cells transfected with prostate-specific antigen RNA stimulate CTL responses against metastatic prostate tumors
}

\author{
Axel Heiser, ${ }^{1}$ Doris Coleman, ${ }^{1}$ Jens Dannull, ${ }^{1}$ Donna Yancey, ${ }^{1}$ Margaret A. Maurice, ${ }^{1}$ \\ Costas D. Lallas, ${ }^{1}$ Philipp Dahm, ${ }^{1}$ Donna Niedzwiecki, ${ }^{2}$ Eli Gilboa, ${ }^{1,3}$ \\ and Johannes Vieweg ${ }^{1}$
}

${ }^{1}$ Cancer Immunotherapy Program, Division of Urology, Department of Surgery,

${ }^{2}$ Department of Biostatistics, and

${ }^{3}$ Experimental Surgery, Department of Surgery, Duke University Medical Center, Durham, North Carolina, USA

Address correspondence to: Johannes Vieweg, Duke University Medical Center, Medical Sciences Research Building, Suite 466, Box 2626, Durham, North Carolina 27710, USA.

Phone: (919) 684-9949; Fax: (919) 681-7414; E-mail: viewe001@mc.duke.edu.

Received for publication October 8, 2001, and accepted in revised form November 20, 2001.

\begin{abstract}
Autologous dendritic cells (DCs) transfected with mRNA encoding prostate-specific antigen (PSA) are able to stimulate potent, $\mathrm{T}$ cell-mediated antitumor immune responses in vitro. A phase I trial was performed to evaluate this strategy for safety, feasibility, and efficacy to induce $T$ cell responses against the self-protein PSA in patients with metastatic prostate cancer. In 13 study subjects, escalating doses of PSA mRNA-transfected DCs were administered with no evidence of dose-limiting toxicity or adverse effects, including autoimmunity. Induction of PSA-specific $\mathrm{T}$ cell responses was consistently detected in all patients, suggesting in vivo bioactivity of the vaccine. Vaccination was further associated with a significant decrease in the log slope PSA in six of seven subjects; three patients that could be analyzed exhibited a transient molecular clearance of circulating tumor cells. The demonstration of vaccine safety, successful in vivo induction of PSA-specific immunity, and impact on surrogate clinical endpoints provides a scientific rationale for further clinical investigation of RNAtransfected DCs in the treatment of human cancer.
\end{abstract}

J. Clin. Invest. 109:409-417 (2002). DOI:10.1172/JCI200214364.

\section{Introduction}

To date, therapeutic options for patients with metastatic prostate cancer are limited to androgen deprivation or systemic chemotherapy. Neither strategy has been proven to extend survival, and, eventually, both will fail in all patients (1). Recently, immunotherapy with dendritic cell-based (DC-based) tumor vaccines has emerged as an alternative therapeutic approach for cancer therapy. Early clinical trials performed in human tumor systems have demonstrated favorable toxicity profiles and therapeutic efficacy in some cancer patients (2-4). DCs, the most potent antigen-presenting cells, are involved in naive $\mathrm{T}$ cell activation and have been shown to be superior to both peptides or proteins alone in stimulating antigen-specific $\mathrm{T}$ cell responses (5). The optimal strategy for tumor antigen delivery to DCs remains one important aspect that clearly deserves further exploration. Antigen can be delivered to DCs in the form of MHC-restricted peptides, proteins, tumorderived antigenic mixtures, or through transfection with genetic materials, each of which greatly influences the pathway and efficacy of T cell activation by DCs.

Recent research suggests that the use of mRNA-transfected DCs represents a new and effective strategy to stimulate cytotoxic Tlymphocyte (CTL) responses in in vitro and in vivo models (6). Indeed, experiments in mice have demonstrated the development of both protective and therapeutic antitumor responses by using mRNA-loaded DC vaccines (7-8). First, for clinical use, the delivery of specific, tumor-associated antigens in an RNA-encoded form appears to be particularly attractive since mRNA can be generated and characterized easier than protein. Second, the RNA template can be further modified to include sequences that may increase the stability and hence the half-live of RNA through polyadenylation or by addition of sequences that may improve antigen presentation (9) by targeting the translated protein to specific MHC compartments. Third, transfection with mRNA carries little, if any, risk of integration into the host genome, leading to subsequent interference with gene expression. Finally, antigens encoded by RNA have the advantage of presenting multiple epitopes for different MHC haplotypes, a fact that expands the scope of vaccination to virtually every cancer patient, independent of their genetic background.

We have shown recently that monocyte-derived DCs cultured from the PBMCs of prostate cancer patients and transfected with prostate-specific antigen (PSA) 
mRNA are capable of stimulating potent antigen-specific CTL responses in vitro (10). We therefore carried out a phase I clinical study to evaluate the safety of administering escalating doses of PSA RNA-transfected DCs in patients with metastatic prostate cancer and to test the feasibility of this approach with respect to vaccine generation. Moreover, we analyzed whether repeated vaccinations with PSA RNA-transfected DCs are capable of stimulating PSA-specific $\mathrm{T}$ cells in the study subjects. Finally, we sought to gain preliminary evidence on a potential clinical benefit as evidenced by serum PSA kinetics and molecular clearance of circulating tumor cells. Here we show that administration of PSA RNA-loaded DCs reliably stimulated the induction of PSA-specific $T$ cell responses without toxicity in all study patients among all dose levels tested, suggesting in vivo bioactivity of the vaccine. Vaccination was further associated with a significant decrease in the log serum PSA slope in six of seven evaluable patients as well as with rapid but transient clearance of circulating tumor cells from peripheral blood of some patients, suggesting some impact on tumor progression. The induction of immunity against the self-protein PSA in association with improvement of surrogate clinical parameters will provide a foundation for ensuing studies analyzing the efficacy of further optimized DC vaccines, preferably performed in a minimal residual disease setting.

\section{Methods}

Vaccine preparation. For DC culture we used techniques described previously (10). Briefly, leukapheresis-derived PBMCs were resuspended in serum-free AIM-V medium (Life Technologies Inc., Grand Island, New York, USA) and incubated in a humidified incubator for 2 hours at $37^{\circ} \mathrm{C}$ to allow plastic adherence. The semiadherent cell fraction was used for DC culture by incubation in serum-free AIM-V medium supplemented with rhIL-4 $(500 \mathrm{U} / \mathrm{ml})$ and rhGM-CSF $(800 \mathrm{U} / \mathrm{ml})(\mathrm{R} \& \mathrm{D}$ Systems Inc., Minneapolis, Minnesota, USA). After 7 days of culture, cells were harvested and characterized to ensure they met the typical phenotype of immature DCs: $\mathrm{CD}^{-}$, CD14-, CD16-/CD56-, CD19-, MHC I+ ${ }^{+}, \mathrm{MHC} \mathrm{II}^{+}$, $\mathrm{CD} 40^{-}, \mathrm{CD}^{\circ} 6^{\text {low }}, \mathrm{CD} 80^{\text {low }}, \mathrm{CD} 83^{-}$. PSA RNA was generated from the plasmid pGEM4/PSA/A64 as described previously (10). Transfection of DCs with PSA RNA was performed by simple coincubation with DCs. Briefly, DCs were washed twice in PBS, counted, and spun for 10 minutes. Subsequently, DCs were resuspended at a concentration of $10^{7}$ cells $/ \mathrm{ml}$ in AIM-V medium and coincubated for 45 minutes with $15 \mu \mathrm{g} / \mathrm{ml}$ RNA. For use as targets, RNA-pulsed DCs were washed once after antigenic stimulation and incubated overnight to allow expression and presentation of PSA antigen.

Patient eligibility. Treatment of patients with PSA RNA-transfected DCs was performed after informed consent on an institutional review board-approved protocol. Patients with confirmed metastatic prostate cancer, clinical stages $\mathrm{D}_{1}-\mathrm{D}_{3}$, were eligible for this study with the following restrictions: serum PSA levels greater than $4.0 \mathrm{ng} / \mathrm{dl}$, Karnofsky performance status (KPS) greater than $70 \%$, estimated life expectancy more than 6 months, white blood cells greater than or equal to $3,000 / \mathrm{mm}^{3}$, platelets greater than or equal to $100,000 / \mathrm{mm}^{3}$, serum creatinine less than $2.5 \mathrm{mg} / \mathrm{dl}$, and bilirubin less than $2 \mathrm{mg} / \mathrm{dl}$. Patients had to have recovered from all toxicities related to any previous therapy and not have received any chemotherapy, radiation therapy, or immunotherapy for at least 6 weeks before entry into the trial. In patients treated with hormone therapy, evidence of appropriate testosterone suppression was obtained before study entry.

Dose and immunization schedule. PSA RNA-transfected DCs were administered at three escalating dose levels with the highest dose to be tested corresponding to the largest number of DCs that can be generated from mononuclear cells isolated by a routine leukapheresis. Dose escalation was performed through an intravenous route using three vaccination cycles with $1 \times 10^{7}$ (low dose), $3 \times 10^{7}$ (medium dose), or $5 \times 10^{7}$ (high dose) cells applied at study weeks 2,4 , and 6 . To optimize vaccination a concomitant dose of $10^{7}$ cells was given intradermally at each vaccination cycle. A second leukapheresis was performed 2 weeks after the last dose to obtain large numbers of cells for immunological monitoring.

Evaluation of immune status. Enzyme-linked immunosorbent spot assay (ELISPOT) was performed using unstimulated PBMCs obtained from baseline and postvaccination leukapheresis. PBMCs were cultured overnight with either PSA, kallikrein, or keyhole limpet hemocyanin $(\mathrm{KLH})$ protein at $5 \mu \mathrm{g} / \mathrm{ml}$ (Sigma Chemical Co., St. Louis, Missouri, USA). After blocking wells with RPMI-1640 medium supplemented with $10 \%$ FCS (complete medium), $5 \times 10^{5}$ cells in 100 $\mu \mathrm{l}$ complete medium were added to each well of flatbottomed 96-well nitrocellulose plates (MultiScreenIP; Millipore Corp., Bedford, Massachusetts, USA) precoated with $2 \mu \mathrm{g} / \mathrm{ml}$ of IFN- $\gamma$ capture Ab (Endogen Inc., Woburn, Massachusetts, USA). Plates were incubated for 20 hours at $37^{\circ} \mathrm{C}$, and after washing, biotinylated IFN- $\gamma$ detection $\mathrm{Ab}(0.2 \mu \mathrm{g} / \mathrm{ml}$; Endogen Inc.) was added to each well. Cells were incubated for an additional 2 hours at room temperature, then incubated with streptavidin-alkaline phosphatase $(1 \mu \mathrm{g} / \mathrm{ml}$, Sigma Chemical Co.) and developed with substrate (Kirkegaard \& Perry Laboratories, Gaithersburg, Maryland, USA). After washing, spots were counted under the microscope. For ${ }^{51} \mathrm{Cr}$ cytotoxicity assays, the $\mathrm{T}$ cell-enriched nonadherent fraction of PBMCs obtained following the plastic adherence step was used for CTL generation. Nonadherent PBMCs were cultured in RPMI cell culture medium supplemented with $20 \mathrm{U} / \mathrm{ml}$ human IL-2 and $10 \mathrm{ng} / \mathrm{ml}$ human IL-7 (R\&D Systems Inc., Minneapolis, Minnesota, USA). Cells were stimulated twice with pulsed autologous DCs at a stimulator to effector ratio of 1:10. After 16 days of culture, effector cells were harvested without further separation and used in microcytotoxicity assays. Target cells were labeled with ${ }^{51} \mathrm{Cr}$ in saline 
solution $\left(\mathrm{Na}_{2}\left[{ }^{51} \mathrm{Cr}\right] \mathrm{O}_{4}\right.$; NEN Life Science Products Inc., Boston, Massachusetts, USA) by incubation of $2 \times 10^{6}$ transfected DCs in $1 \mathrm{ml}$ RPMI with $100 \mu \mathrm{Ci}$ of ${ }^{51} \mathrm{Cr}$ for 1 hour at $37^{\circ} \mathrm{C} / 5 \% \mathrm{CO}_{2}$. After three washes, $5 \times 10^{3}$ ${ }^{51} \mathrm{Cr}$-labeled targets and serial dilutions of effector cells at various effector/target ratios were incubated in 200 $\mu \mathrm{l}$ of RPMI in 96-well U-bottom plates. These plates were incubated for 6 hours at $37^{\circ} \mathrm{C} / 5 \% \mathrm{CO}_{2}$. Then, supernatants were harvested, and the released ${ }^{51} \mathrm{Cr}$ was measured with a scintillation counter. Spontaneous release was less than $15 \%$ of the total release by detergent in all assays. Standard deviation of the means of triplicate wells was less than $5 \%$. Calculation of lytic units for the expression of cell-mediated cytotoxicity was calculated as described previously (11).

Biostatistical assessment of serum log slopes PSA. Serum PSA levels were collected on seven patients who completed immunotherapy at time points before and after initiation of treatment. The linear regression model was used to obtain estimates of the change of serum PSA over time. Two curves were fitted for each patient based on the data before starting treatment and after treatment, respectively. The difference between the preand post-treatment slope estimates was computed for each patient and compared using the two-sided Wilcoxon matched-pairs signed rank test.

Enumeration of circulating tumor cells using real-time PCR. Total RNA was extracted from $20 \mathrm{ml}$ of peripheral blood $\left(2 \times 10^{7}\right.$ PBMCs) using the RNeasy Maxi kit (QIAGEN Inc., Valencia, California, USA) according to the manufacturer's protocol. For cDNA synthesis, $1 \mu \mathrm{g}$ of total RNA was reverse-transcribed using the Gold RNA PCR Core kit (Perkin-Elmer Applied Biosystems, Foster City, California, USA). Amplified cDNA was purified and quantitated by spectrophotometry. Copy numbers were calculated using the molecular weight of each individual gene amplicon. RT-PCR reactions of cDNA samples and cDNA standards were performed in a total volume of $40 \mu \mathrm{l}$ of $1 \times$ TaqMan Master mix (Perkin-Elmer Applied Biosystems) containing $0.2 \mu \mathrm{M}$ of each primer and $0.625 \mu \mathrm{M}$ of probe, respectively. Thermal cycler parameters included 2 minutes at $50^{\circ} \mathrm{C}, 10$ minutes at $95^{\circ} \mathrm{C}$, followed by 40 cycles of amplification with 15 seconds of denaturation at $95^{\circ} \mathrm{C}$ and 1 minute of annealing/extension at $60^{\circ} \mathrm{C}$. Measurement of gene expression was performed using the ABI Prism 7700 Sequence Detection System. TaqMan probes used in our assays were labeled at the $5^{\prime}$ end with a reporter dye and at the $3^{\prime}$ end with a quencher molecule. The following probes were used: PSA, 5'-JOE (6-carboxy-4, 5-dichloro-2, 7-dimethoxyfluorescein)-CAAAAGCACCTGCTCGGGTGATTC-TAMRA (6-carboxy-N,N, $\mathrm{N}^{\prime}, \mathrm{N}^{\prime}$ tetramethylrhodamine)-3'; EpCAM, 5'-JOE-AGTGCAGTCCGCAAACTTTTACTATCATAAGGTTTT-TAMRA-3'. Amplification of the respective gene product was performed with the following primer pairs: PSA forward, 5 -ACCCTCAGAAGGTGACCAAGT-3' and PSA ${ }^{\prime}$ reverse, 5'-TGAAGCACACCATTACAGACAA-3'; EpCAM forward $^{\prime} 5^{\prime}$-TCATTGAACTAA-
AACACAAAGCAAGA-3' and EpCAM reverse, $5^{\prime}$-CGCGTTGTGATCTCCTTCTG-3'. Real-time monitoring of fluorescence emission allowed definition of the threshold cycle during the exponential phase of amplification. Standard curve extrapolation of copy number was performed for each gene as well as the endogenous reference $\beta$-actin (12). Normalization of samples was performed by dividing the copies of the gene of interest by copies of the reference gene.

\section{Results}

Vaccine and patient characteristics. Monocyte-derived DCs were generated from peripheral blood precursors cultured in low-protein medium solely supplemented with the cytokines IL-4 and GM-CSF. Immature DCs expressing the phenotype $\mathrm{MHCI}^{+}, \mathrm{MHC} \mathrm{II}^{+}, \mathrm{CD}^{1} 0^{\text {low }}, \mathrm{CD} 86^{\text {low }}$, $\mathrm{CD}^{-} 3^{-}, \mathrm{CD}^{-}, \mathrm{CD} 14^{-}, \mathrm{CD} 16 / \mathrm{CD}^{-} 6^{-}, \mathrm{CD}^{-} 9^{-}$were used for subsequent RNA transfection, carried out by short-

a
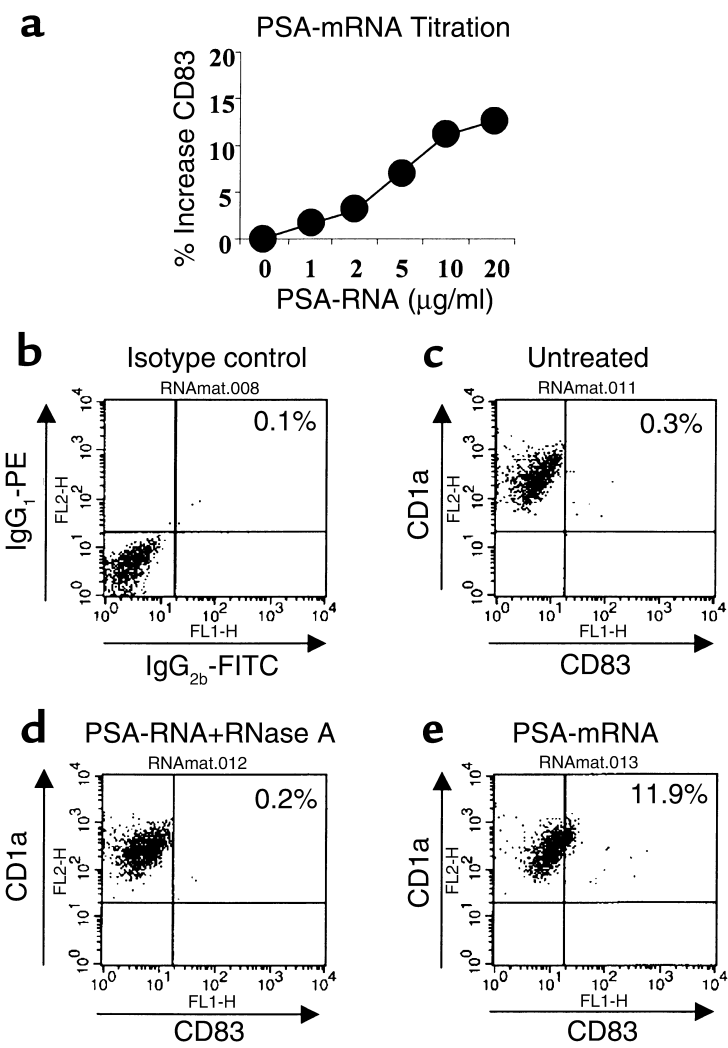

Figure 1

Incubation of immature DCs with PSA RNA provides a maturation stimulus. (a) Upregulation of CD83 expression by immature DCs in the presence of increasing PSA mRNA concentrations. (b-e) Immature DCs were incubated for 45 minutes in the presence of $20 \mu \mathrm{g} / \mathrm{ml}$ of PSA mRNA and analyzed after 48 hours by flow cytometry for expression of the DC-specific marker CD1a and the DC maturation marker CD83, respectively. (b) Staining with isotype immunoglobulins $\lg _{2 b}-$ FITC and IgG 1 -PE (isotype control). (c) DCs incubated in the absence of PSA mRNA (untreated). (d) DCs incubated with PSA mRNA that had been treated for 1 hour at $37^{\circ} \mathrm{C}$ with $10 \mu \mathrm{g} / \mathrm{ml}$ RNase A (PSA-RNA + RNase A), and (e) DCs incubated in the presence of PSA mRNA. One representative experiment out of three independent experiments is presented. 
Table 1

Characteristics of patients enrolled, including status before vaccination and response to vaccination

\begin{tabular}{|c|c|c|c|c|c|c|c|c|c|c|c|c|c|c|}
\hline \multirow[t]{2}{*}{$\begin{array}{l}\text { Patient } \\
\text { ID no. }\end{array}$} & \multirow[t]{2}{*}{ Age } & \multirow{2}{*}{$\begin{array}{l}\text { Karnofsky } \\
\text { index }\end{array}$} & \multirow{2}{*}{$\begin{array}{c}\text { Onset } \\
\text { stage } D_{3} \\
\text { (months) }\end{array}$} & \multirow{2}{*}{$\begin{array}{l}\text { Previous } \\
\text { therapy }\end{array}$} & \multirow[t]{2}{*}{ Stage } & \multirow{2}{*}{$\begin{array}{l}\text { PSA } \\
\text { (study } \\
\text { entry) }\end{array}$} & \multirow{2}{*}{$\begin{array}{c}\text { Metastases } \\
\text { (study } \\
\text { entry) }\end{array}$} & \multirow{2}{*}{$\begin{array}{l}\text { Assigned } \\
\text { dose } \\
\text { level }\end{array}$} & \multirow[t]{2}{*}{$\begin{array}{c}\text { Actual } \\
\text { dose }\end{array}$} & \multicolumn{2}{|c|}{$\begin{array}{l}\text { Log PSA } \\
\text { slope }\end{array}$} & \multirow{2}{*}{$\begin{array}{c}\text { T cell } \\
\text { frequencies } \\
\text { (ELISPOT post) }\end{array}$} & \multirow{2}{*}{\multicolumn{2}{|c|}{$\begin{array}{l}\text { Cytotoxicity } \\
\text { pre post } \\
\text { (lytic units }\end{array}$}} \\
\hline & & & & & & & & & & pre & post & & & \\
\hline $\mathrm{JH}-01$ & 76 & 85 & 12 & $\mathrm{XRT}^{1}$ & $\mathrm{D}_{3}$ & 143 & $\mathrm{LN}$ & Low & Low & 0.032 & $\mathrm{SCT}$ & ND & 71 & 103 \\
\hline JD-02 & 60 & 90 & 24 & $\mathrm{RP}$ & $\mathrm{D}_{3}$ & 48 & $\mathrm{BN}$ & Low & Low & 0.015 & 0.019 & 32 & 73 & 139 \\
\hline TL-03 & 62 & 100 & 24 & None & $\mathrm{D}_{3}$ & 6 & $\mathrm{BN}$ & Low & Low & 0.069 & 0.012 & 51 & 71 & 162 \\
\hline WP-05 & 64 & 90 & 16 & $\mathrm{RP} / \mathrm{XRT}^{2}$ & $\mathrm{D}_{3}$ & 18 & $\mathrm{BN}$ & Med & Med & 0.047 & 0.090 & 50 & 79 & 170 \\
\hline EA-06 & 51 & 90 & 20 & $\mathrm{XRT} T^{1,2}$ & $\mathrm{D}_{3}$ & 267 & $\mathrm{BN} / \mathrm{LN}$ & Med & Med & 0.188 & $\mathrm{XRT}^{3}$ & 97 & 63 & 161 \\
\hline $\mathrm{VH}-07$ & 58 & 90 & 22 & $\mathrm{XRT}{ }^{1}$ & $D_{3}$ & 220 & $\mathrm{BN}$ & High & Med & 0.027 & -0.011 & 68 & 106 & 130 \\
\hline Jl-08 & 49 & 90 & - & None & $\mathrm{D}_{2}$ & 35 & $\mathrm{BN}$ & Med & Med & 0.071 & $\mathrm{SCT}$ & ND & 68 & 90 \\
\hline JB-09 & 70 & 90 & 15 & $\mathrm{RP} / \mathrm{XRT}^{2}$ & $\mathrm{D}_{3}$ & 238 & $\mathrm{BN}$ & High & High & 0.180 & 0.039 & 198 & 71 & 143 \\
\hline $\mathrm{GH}-10$ & 63 & 90 & - & $\mathrm{RP} / \mathrm{O}$ & $\mathrm{D}_{1}$ & 13 & LN & High & High & 0.017 & $\mathrm{HB}$ & ND & 39 & 66 \\
\hline TH-11 & 67 & 90 & 16 & $\mathrm{RP} / \mathrm{XRT}^{2}$ & $D_{3}$ & 118 & $\mathrm{BN} / \mathrm{LN}$ & High & High & 0.012 & $\mathrm{HB}$ & 148 & ND & ND \\
\hline JM-13 & 62 & 90 & 11 & None & $\mathrm{D}_{3}$ & 8 & $\mathrm{BN}$ & High & High & 0.020 & 0.013 & 261 & 71 & 182 \\
\hline $\mathrm{AH}-15$ & 73 & 90 & 23 & None & $D_{3}$ & 73 & BN/LN & High & High & 0.029 & $\mathrm{XRT}{ }^{3}$ & ND & ND & ND \\
\hline WV-16 & 68 & 90 & 8 & None & $D_{3}$ & 10 & $B N / L N$ & High & High & 0.080 & 0.053 & ND & ND & ND \\
\hline
\end{tabular}

Low dose: $1 \times 10^{7}$ cells intravenously, $1 \times 10^{7}$ cells intradermally for three cycles; medium dose: $3 \times 10^{7}$ cells intravenously, $1 \times 10^{7}$ cells intradermally for three cycles; high dose: $5 \times 10^{7}$ cells intravenously, $1 \times 10^{7}$ cells intradermally for three cycles. Pre- or post-vaccination treatment: $\mathrm{XRT}^{1}$, primary irradiation; $\mathrm{XRT}^{2}$ adjuvant irradiation; $\mathrm{XRT}^{3}$, local (palliative) irradiation for painful bony metastases; RP, radical prostatectomy; O, orchiectomy; SCT, systemic chemotherapy; HB, herbal supplements; none, no therapy; ND, not determined. Metastases: LN, lymphadenopathy; BN, bony metastases.

term coincubation of "naked" PSA mRNA and the cultured DCs (10). Incubation of immature DCs with PSA mRNA (endotoxin $<0.05 \mathrm{EU} / \mathrm{ml}$ ) resulted in a modest, but consistent increase of the cell surface markers CD83 (Figure 1a) and CD86 (data not shown) in an mRNA dose-dependent fashion. CD83 expression was abolished upon treatment with RNase (Figure 1d) but not DNase (data not shown). Consistent with these findings, immature DCs incubated with total cellular RNA (isolated from tumor cells) also exhibited upregulation of CD83 expression at similar levels providing additional evidence that maturation was mediated by mRNA and not by contaminating DNA or endotoxin (data not shown).

Sixteen patients with histologically proven, metastatic prostate cancer stages $\mathrm{D}_{1}-\mathrm{D}_{3}$ were enrolled in this study; their detailed characteristics are shown in Table 1. Thirteen patients were fully evaluable for analysis since three patients required withdrawal from study due to rapid and symptomatic tumor progression during the vaccination phase $(n=2)$ and an acute septic event unrelated to the vaccine $(n=1)$. Since PSA was used as a candidate antigen in this study, only patients with PSAoverexpressing tumors (PSA > $4 \mathrm{ng} / \mathrm{dl}$ ) were enrolled. Pretreatment serum PSA levels varied widely among the study subjects harboring diffuse bony and/or lymphatic metastases (range, $6 \mathrm{ng} / \mathrm{dl}-267 \mathrm{ng} / \mathrm{dl}$ ).

Vaccine safety and feasibility of vaccine generation. We were able to escalate the DC dose administered intravenously to the proposed maximal achievable dose (three cycles using $5 \times 10^{7}$ cells at each cycle). This cell number chosen for the high dose level was based on the maximum number of DCs that could be reproducibly generated ex vivo from PBMCs of prostate cancer patients obtained during a single leukapheresis. Three subjects received three cycles of $10^{7}$ PSA RNA-transfected DCs (low dose) given as an intravenous infusion at each treatment cycle (study week 2, 4, and 6). Three subjects were treated at a medium (three cycles of $3 \times 10^{7} \mathrm{DCs}$ ), and six subjects at a high dose level (three cycles of $5 \times 10^{7} \mathrm{DCs}$ ). For all dose levels a concomitant intradermal injection of $10^{7} \mathrm{DCs}$ was given during each treatment cycle. Vaccination was well tolerated and no major (greater than grade I) toxicity during all vaccine cycles was observed in any subject. Four subjects demonstrated grade I toxicities in the form of body temperature elevations accompanied by flulike symptoms. Four subjects exhibited grade I injection site reactions consisting of inflammatory erythema lasting 48-72 hours. There were no treatment-related hematologic, hepatic, renal, or neurological toxicities. In one subject (JB-09) we noted a transient elevation of antinuclear $\mathrm{Ab}$ titers and rheumatoid factor (grade 1) following the final vaccination cycle, which spontaneously returned to baseline within a 2 -week period. In 12 of 13 subjects the assigned cell dose could be generated from leukapheresis-derived DCs. One subject within the highdose group (VH-07) was treated at a medium-dose level due to insufficient numbers of DCs that could be retrieved from cryopreserved and reconstituted samples. This subject was subsequently replaced and an additional patient (WV-16) was enrolled and successfully treated at the high dose level. Cumulatively, these data suggest low-toxicity profiles of the PSA RNA-transfected DCs throughout all dose levels tested. They also demonstrate the feasibility and reproducibility of generating large numbers of PSA RNA-transfected DCs from subjects with metastatic prostate cancer using clinically compatible and safe cell manufacturing procedures.

Cellular response to vaccine therapy. To determine whether vaccination with PSA RNA-transfected DCs is capable of stimulating $\mathrm{T}$ cell responses in the treated patients, we first analyzed and compared the numbers of PSA-specific T cells directly from pre- and posttherapy PBMC samples using the IFN- $\gamma$ ELISPOT assay. PBMCs obtained at baseline and following the 
third vaccination (study week 6) were cultured with PSA protein overnight and analyzed for the expression of intracellular IFN- $\gamma$. PSA protein, but not peptide, was used as an antigenic stimulus since we sought to provide analysis for all patients enrolled (who had different HLA haplotypes). Previous experiments had shown that an overnight pulse with PSA protein onto $5 \times 10^{5}$ PBMCs seeded per well generates optimal results with respect to the detection efficacy of single spot-forming $\mathrm{T}$ lymphocytes. As shown in Figure 2, the numbers of IFN- $\gamma$-secreting cells was low or undetectable when pretherapy PBMC samples were analyzed, whereas after vaccination all nine patients analyzed had measurable, PSA-reactive $\mathrm{T}$ cells at frequencies ranging from $1 / 10,000$ to $1 / 2,000$. No reactivities were observed against negative control proteins such as human serum kallikrein (KK) or KLH (data not shown) in any PBMC sample obtained from study subjects before or after vaccination. Although there was significant patient-to-patient variability in the magnitude of the $T$ cell responses observed among all dose levels, this increase appeared to be more pronounced in subjects treated at the medium- and highdose levels, suggesting the induction of PSA-reactive $T$ cells in a cell dose-dependent manner.

The consistently observed increase in the numbers of detectable PSA-specific T cells following vaccination is remarkable in view of the fact that analysis was performed directly from peripheral blood cells without the need for repeated in vitro restimulations. Importantly, PSA RNA-transfected DCs did not induce $\mathrm{T}$ cell responses against epitopes expressed by KK, a protein that shares significant structural homology with PSA. These results furthermore are consistent with the clinical safety data, suggesting that unwanted cross-reactivities between the PSA-specific T cells and normal housekeeping gene products such as KK may not occur and that therefore harmful autoimmunity may not represent a serious issue with this approach. In this study the use of exogenous PSA protein as a stimulus in the ELISPOT resulted in detection of both PSA-specific CD8 (CTL) and CD4 T cells with a potential bias to increased detection of CD4 T helper lymphocytes. Therefore, in order to analyze the contributions of MHC class I-restricted CD8 T cells on this response we chose to perform standard cytotoxicity assays, which specifically analyze CTL-mediated killing of PSAexpressing target cells. Unfortunately, tumor cells commonly used in these assays as specific targets are either inaccessible or difficult to culture, particularly from prostate cancer patients. Here we used PSA RNA-transfected DCs as surrogate targets in the cytotoxicity assays since previous work has shown that PSA-specific CTLs are capable of recognizing and lysing ${ }^{51} \mathrm{Cr}-$ labeled tumor cells as well as PSA RNA-transfected DCs in an identical manner (10).

Validation of PSA RNA-transfected DCs as antigen-specific targets. To confirm and further extend these observations, PSA RNA-transfected DCs were generated from a HLA-A2 $2^{+}$prostate cancer patient to generate CTLs from PBMCs in vitro. After two restimulation cycles, effector cells were seeded and cocultured with cellular target cells at increasing effector target ratios for 60 minutes, and lytic activities were calculated at each effector to target $(\mathrm{E} / \mathrm{T})$ ratio. The following specific or control targets were used: (a) PSA RNA-transfected DCs, (b) human LNCaP cells (HLA-A2 ${ }^{+}, \mathrm{PSA}^{+}$), (c) T2 cells pulsed with a HLA-A2-restricted PSA peptide (PSA-3), (e) green fluorescent protein (GFP) RNA, or (f) unpulsed T2 cells. Similar to our observations published previously (10), the in vitro-stimulated CTLs were capable of recognizing and lysing PSA RNA-transfected DCs and human LNCaP cells with similar efficacy (data not shown). PSA peptide-pulsed T2 cells were also lysed, albeit less efficiently than the PSA RNA-transfected DCs or LNCaP targets, suggesting that the CTL response was directed against many epitopes present on PSA that encompassed as a subcomponent a response against the single epitope presented by PSA peptide-pulsed T2 cells. No crossreactivities against GFP-expressing DC targets or unpulsed T2 cells were noted.

In summary, these experiments not only demonstrate that PSA RNA-transfected DCs represent effective stimulators of antigen-specific $\mathrm{T}$ cell responses, they also validate their use as antigen-specific targets in CTL assays. This is of particular relevance for immune monitoring of prostate cancer patients since primary prostate tumor cells routinely are either unavailable or difficult to culture, precluding their routine use in CTL assays. We realize that the PSA RNA-transfected DCs used as targets in CTL assays may not accurately mirror the susceptibility of lysis to prostate tumor targets by PSA-specific CTLs since they may fail to reproduce the loss or downregulation of MHC class I molecules

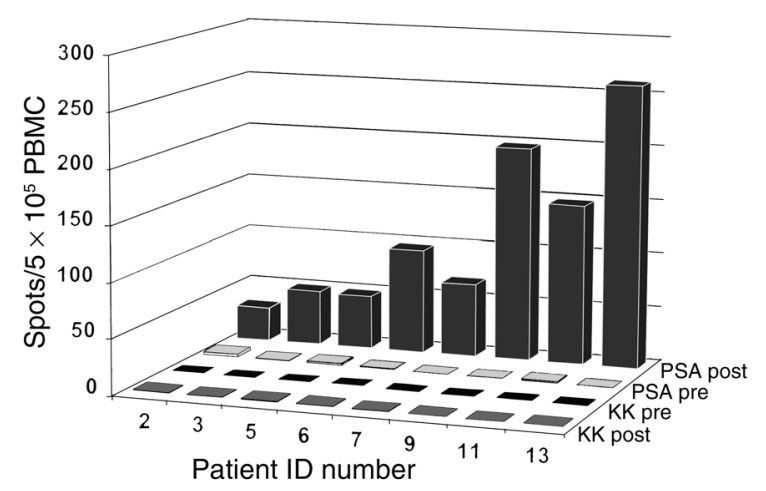

Figure 2

In vivo induction of PSA-specific T cell responses. PBMCs obtained at baseline (PSA, KK pre) and after three vaccination cycles (PSA, KK post) using PSA RNA-transfected DCs were cultured overnight with PSA or kallikrein protein $(5 \mu \mathrm{g} / \mathrm{ml})$. The specific T cell frequencies in each of the evaluable patients treated at a low dose level $\left(10^{7}\right.$ cells; patient 2,3 , and 5$)$, medium dose level $\left(3 \times 10^{7}\right.$ cells; patient 6,7 , and 9$)$, or high dose level $\left(5 \times 10^{7}\right.$ cells; patient 11 and 13$)$ is expressed as the number of spot-forming cells per $5 \times 10^{5}$ PBMCs seeded in each well. 
a

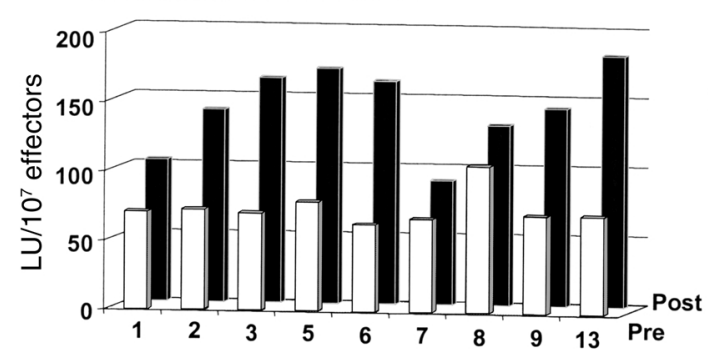

b

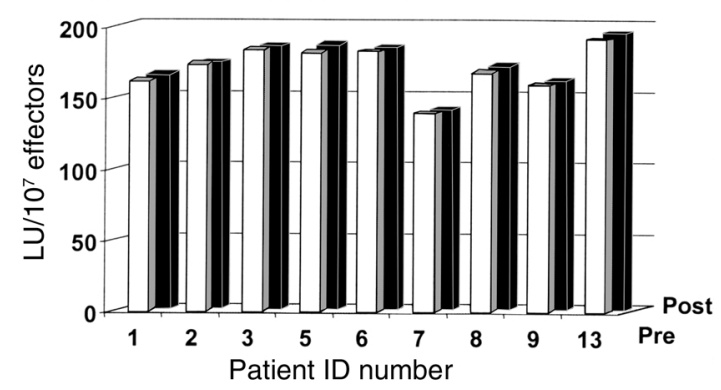

C

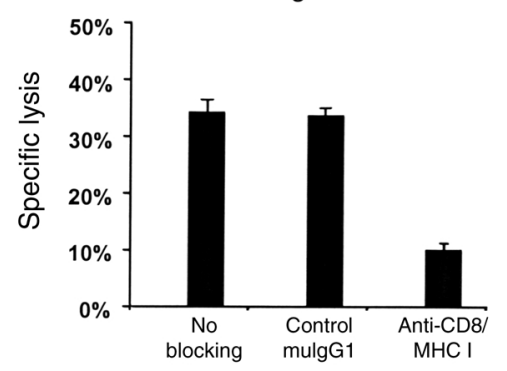

Figure 3

Functional properties of the in vivo-generated PSA-specific CTLs. PBMCs derived from study patients at baseline (white bars) or after immunotherapy (black bars) were stimulated twice with PSA RNA (a) or GFP RNA-transfected DCs (b). Using standard ${ }^{51} \mathrm{Cr}$ microcytotoxicity assays, lytic activities against PSA RNA- or GFP RNA-transfected DC targets were calculated. (c) Experiments in which MHC class I- or CD8-specific mAb's were used to inhibit recognition and lysis of PSA RNA-loaded DC targets. The addition of these mAb but not isotype control mAb (Control mulgG1) during incubation of effector and target cells (40:1 E/T ratio) resulted in significant inhibition of PSAspecific cytolytic activity, suggesting that the observed responses are predominately mediated by MHC class I-restricted CD8 CTL.

often seen in prostate cancers. On the other hand, RNA-transfected DCs may allow us to reliably measure and compare the antigenic content of tumor cells that are available for class I presentation but not necessarily presented by the tumor cell.

Stimulation of PSA-specific CTLs from immunized subjects. We next analyzed the effector function of the in vivo-generated PSA-specific T cells to specifically recognize and lyse PSA-expressing DC targets. Specifically, we tested whether there is an increase in target cell lysis using PSA-specific $T$ cells stimulated from postvaccination PBMCs when compared with pretherapy samples. To generate CTLs, cryopreserved pre- and post-therapy PBMCs were thawed, reconstituted, and stimulated in vitro at the same time using two restimulation cycles with PSA RNA-transfected DCs (Figure 3a). As controls, separate aliquots of pre- or postvaccination PBMC samples were stimulated with DCs transfected with RNA encoding the highly immunogenic GFP and tested against GFP RNA- and PSA RNA-transfected DC targets (Figure $3 b$ ). As shown in Figure 3 , $a$ and $b$, the restimulated PBMCs resulted in a significant increase in PSA-specific, but not GFP-specific lytic CTL activity in all nine patients analyzed after vaccination (black bars) when compared with pretreatment lytic activity against PSA targets (white bars). Even a single restimulation cycle resulted in a modest, albeit significant, increase in the lytic levels against PSA RNA-transfected DC targets (data not shown), whereas GFP-specific CTLs generated from baseline or posttherapy samples demonstrated no differences in their lytic activities after therapy (Figure $3 b$ ). To determine whether the observed increase in the cytotoxic activity is mediated by MHC class I-restricted CD8 T cells, we performed blocking experiments using monoclonal $\mathrm{Ab}$ 's added during the incubation phase of effector and target cells (E/T ratio of 40:1) following two restimulation cycles (Figure 3c). The addition of a CD8 or MHC class I (data not shown) $\mathrm{mAb}$, but not a control $\mathrm{Ab}$, resulted in significant inhibition of the PSA-specific cytolytic activity suggesting that the $T$ cells expanded during restimulation were predominately CD8 effector cells and responsible for the killing of the PSA-expressing targets. Cumulatively, the data shown in Figures 2 and 3 provide evidence that vaccination with PSA RNA transfected DCs may stimulate PSA-specific T cells, including CD8 $\mathrm{T}$ cells in vivo, the latter of which are capable of specifically recognizing and lysing cellular targets expressing PSA.

Changes in serum PSA levels in patients receiving active immunotherapy with PSA RNA-transfected DCs. Although this exploratory trial was not designed to detect clinical responses, we monitored serum PSA levels of each study candidate at baseline, during, and after active immunotherapy as a surrogate marker for clinical response. As described by Slovin and coworkers (13), we calculated and compared serum log slopes PSA (PSA velocities) before and after completion of the three vaccination cycles from seven evaluable subjects. The other six subjects were excluded from analysis due to the emergence of new or progression of preexisting painful bony metastases during follow-up requiring palliative irradiation $(n=2)$ or due to patient noncompliance with intake of herbal compounds with intrinsic estrogenic activity, thus impacting on PSA levels $(n=4)(14)$. Although in six of the seven evaluable patients the absolute serum PSA concentrations continued to rise during follow-up, a decrease of the serum PSA levels was observed in one patient (VH-07) consistent with a minor response according to the NIH response criteria (Figure 4a). Interestingly, when pre- and post-treatment PSA velocities were calculated and compared, a significant decrease of the post-treatment log slope PSA was noted 
in five additional study patients (patients 3, 5, 9, 13, and 16) (as exemplified in Figure 4b), while in one patient (JD-02) post-therapy PSA velocities remained statistically unchanged (Figure 4c). It may be argued that the observed decreases of serum PSA slopes are, at least in part, due to induction of a vaccine-mediated humoral immune response, analyses aimed to detect PSA-specific Ab's in the serum of study subjects did not reveal significant differences among pre- and postvaccination (2 weeks after the last treatment cycle) titers of PSA Ab in all patients (data not shown). In conclusion, the estimated log slope PSA decreased in six of seven evaluable patients after initiation of immunotherapy. Although, at this point, the true clinical significance of these findings remains unclear and awaits further correlation with standard clinical outcome parameters, it appears that vaccination with PSA RNA-transfected DCs is not only capable of inducing potent $\mathrm{T}$ cell responses in prostate cancer patients, but may also result in significant decreases of serum PSA velocities, suggesting some, albeit modest, impact on tumor progression.

Real-time PCR to detect hematogenous micrometastases. Recent research has introduced the novel concept that the vaccine-induced elimination of tumor cells circulating in the peripheral blood of cancer patients may predict their clinical course or may even correlate with improved survival (15). Here we tested whether vaccination with PSA RNA-loaded DCs can stimulate antitumor effects, which result in the elimination of tumor cells shed from the primary tumor into the systemic circulation. We used real-time PCR to quantitatively assess the kinetics of circulating tumor cells at baseline, during, and after vaccination in three patients on trial using primers and probes specific for PSA or the epithelial marker EpCAM (16).

PSA or EPCAM mRNA was amplified from total RNA extracted from $10^{7} \mathrm{PBMCs}$, the corresponding copy numbers were quantitated, and those specific for the reference gene $\beta$-actin were quantitated and subsequently normalized to $\beta$-actin RNA copy numbers amplified from the same PBMC sample. To further improve the sensitivity of this assay, we also determined the average mRNA copy number of each marker amplified from $10^{7}$ PBMCs of ten age-matched healthy male volunteers. Following normalization to $\beta$-actin, an absolute copy number greater than 0.25 for PSA or greater than 70 for EpCAM was considered as significant elevation above background (Figure 5, a and b; dotted lines). Since sufficient numbers of PBMCs were available for only a subset of patients to allow longitudinal tumor cell monitoring requiring peripheral blood sampling every 2 weeks, we were able to analyze only three volunteering patients enrolled at the medium (WP-05) or high-dose level (JM-13, WV-16). Among these patients, two had significant elevations in the absolute copy number of PSA mRNA, but not EpCAM mRNA prior to therapy (WP-05, JM-13), while in the remaining patient (WV-16) only EpCAM but not PSA mRNA copy numbers were above those of healthy volunteers prior to the study. As exemplified in Figure 5, a and $b$, the number of circulating tumor cells in each patient declined below healthy volunteer background levels upon initiation of treatment, a phenomenon that was not reflected by the consistently rising serum PSA levels. In the patients analyzed, clearance of circulating tumor cells was of relatively short duration and significant numbers of cells became detectable at various intervals during follow-up ( 3 weeks in patient JM-13, 8 weeks in patients WV-16 and WP-05). In two patients (WP-03 and JM-13), the reappearance of circulating tumor cells was associated with radiologically documented progression of disease, while the other patient (WV-13), who remains in molecular remission (levels of circulating tumor cells above background but still below pretreatment levels), demonstrated a stable clinical course. We have made similar observations by analyzing the kinetics of circulating
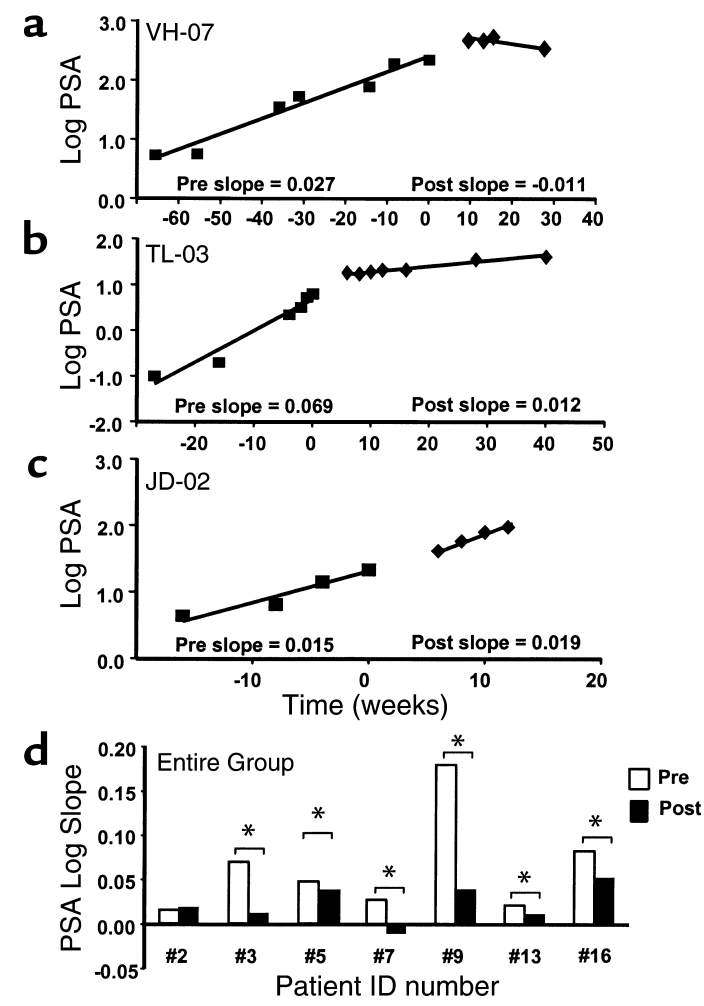

\section{Figure 4}

PSA velocities after vaccination with PSA RNA-transfected DCs. Pretherapy (pre slope) and post-treatment (post slope) serum PSA kinetics of study candidates vaccinated with PSA RNA-transfected DCs were calculated as described previously (13). A linear regression model was used to obtain estimates on the change of serum PSA over time, and the differences between pre- and post-therapy log slope estimates were computed for each patient. (a-c) The results on the serum PSA kinetics in all seven patients who were eligible for analysis before (pre) and after (post) vaccine therapy. In one of seven patients available for analysis (a), a decrease in the log slope PSA was calculated upon initiation of therapy. In five of the seven evaluable patients we could demonstrate a significant reduction of the log slope PSA (patient 3, 5, 7, 9, 10) (b), whereas one patient (patient 2) exhibited unchanged (c) PSA velocities after treatment with PSA RNA-pulsed DCs. (d) Pre- (white bars) and post-therapy (black bars) log slope PSA values for the entire group. Asterisk indicates statistically significant differences between the pre- and post-treatment slope. 

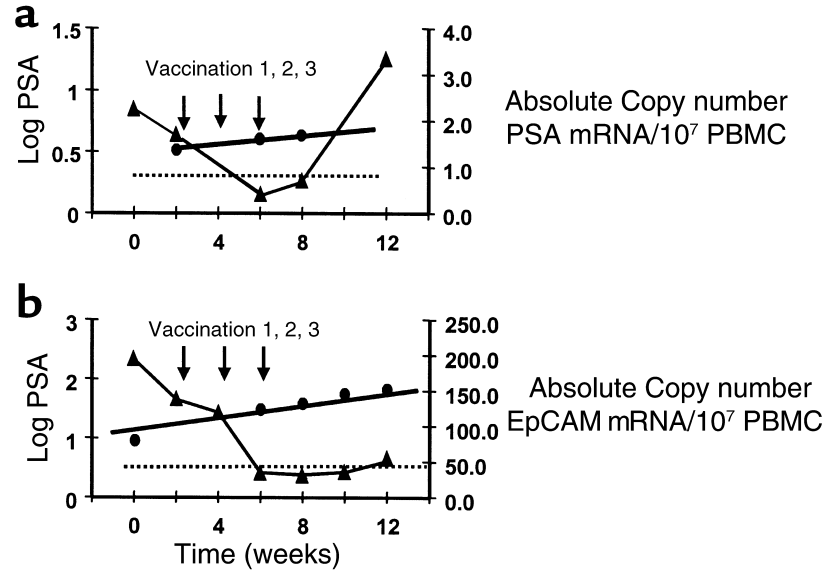

\section{Figure 5}

Molecular clearance of circulating tumor cells after vaccination. Realtime PCR was used to quantitatively assess the kinetics of circulating tumor cells at baseline (week 0), during vaccination (week 2, 4, 6), and after vaccination (weeks $8,10,12$ ) in three study patients using primers and probes specific for PSA (a) or the epithelial marker EpCAM (b). PSA or EpCAM mRNA was amplified from total RNA extracted from $10^{7}$ PBMCs, and the corresponding copy numbers were quantitated and subsequently normalized to $\beta$-actin RNA copy numbers amplified from the same PBMC sample. To further improve the sensitivity of this assay, we also determined the average mRNA copy number of each marker amplified from PBMCs of ten agematched healthy male volunteers (dotted lines). In three patients analyzed, vaccination with PSA RNA-transfected DCs led to a complete but temporary clearance of blood-borne circulating tumor cells, an effect that was not predicted by serum PSA levels.

tumor cells in prostate cancer patients following chemotherapy or hormonal therapy demonstrating a molecular reduction or clearance followed by rapid increase upon cessation of therapy (unpublished observations). In these studies we could consistently detect circulating tumor cells in all metastatic prostate cancer patients using real-time PCR with primers and probes for PSA or for EPCAM.

In summary, these data suggest that three vaccination cycles with PSA RNA-transfected DCs are associated with the transient elimination of tumor cells from the peripheral bloodstream of some prostate cancer patients. The kinetics of the circulating tumor cell levels observed in these patients may suggest that this vaccination protocol (using this route and frequency of vaccination) induces a significant albeit temporary vaccine effect, which may be enhanced by additional vaccination cycles or by boosting. Although, at this point, the prognostic significance of enumerating circulating tumor cells is uncertain and awaits further validation, changes in their levels may indeed allow predictions on the magnitude and duration of treatment response and may assist us to further improve vaccination strategies.

\section{Discussion}

We have shown previously that PSA RNA-transfected DCs represent an effective strategy to stimulate primary $\mathrm{T}$ cell responses from PBMCs of prostate cancer patients in vitro (10). Unlike peptide-based vaccines, which are limited in their therapeutic use to certain patient subsets, the use of DCs transfected with RNAencoded antigens reproducibly allows stimulation of PSA-specific CTLs from PBMCs of virtually all prostate cancer patients independent of their MHC composition. Furthermore, we have shown that it is possible to use total (17) or amplified tumor RNAloaded (18) DCs to stimulate even more potent and polyclonal immune responses when compared with DCs transfected with RNA encoding single antigens or pulsed with antigenic peptides.

As a first step in the clinical development of RNAbased DC vaccines we initiated a phase I trial designed to establish the safety of administering DCs transfected with PSA mRNA and provide data on the feasibility of generating PSA RNA-transfected DCs from PBMCs of cancer patients on a large scale. We further sought to obtain preliminary information on the immune responses induced by vaccination and to develop a defined model system allowing further refinements of this strategy in prostate cancer patients. While several recent reports have indicated that it is possible to induce humoral or cell-mediated $\mathrm{T}$ cell responses (19-20) against PSA in a clinical trial setting, they also support the crucial need for effective antigen-presenting platforms to further enhance PSA-specific immunity. Two recent clinical trials have used DCs pulsed with prostate membrane antigen-specific peptides (21) or with a fusion protein consisting of prostatic acid phosphatase linked to GM-CSF (22) and provided evidence for both immunological and clinical responses in patients with metastatic prostate cancers.

Our study using PSA RNA-transfected DCs clearly demonstrates the safety and feasibility of this approach with no apparent adverse events or dose-limiting toxicities encountered in all subjects on protocol. Only four study candidates were withdrawn from study or substituted either due to rapid disease progression $(n=3)$ or due to failure to produce the vaccine $(n=1)$, further demonstrating the broad applicability of this strategy even in unfavorable subjects carrying metastatic disease. Although this study was not designed to assess treatment efficacy, we provide several lines of evidence that repeated vaccination with PSA RNA-transfected DCs also provides in vivo bioactivity by stimulating PSA-specific $\mathrm{T}$ cells, including MHC class I-restricted CD8 cytotoxic T cells, in prostate cancer patients. As shown in Figures 2 and 3, all patients on trial exhibited increases in PSA-specific $\mathrm{T}$ cell responses as a result of vaccination. In this study, immature DCs were used for RNA pulsing, since it was shown that only DCs of the immature phenotype are competent for successful RNA uptake, processing, and antigen presentation (9). Recent research has indicated that immature DCs may have only modest antigen-presenting or even tolerizing function, while only mature DCs are capable of potently stimulating $\mathrm{T}$ cells in vivo (23). As shown in Figure 1, trans- 
fection with naked PSA mRNA provides a partial maturation stimulus to DCs leading to increased expression of the activation markers CD83 and CD86. These finding are consistent with other recent reports suggesting that RNA alone may deliver a maturation signal transforming DC into a semimature state (24), possibly facilitating the full maturation of the injected DC. We therefore believe that the RNA-transfected DCs used in this clinical study were already committed to maturation and thus capable of priming $T$ cell reactivities in vivo, notwithstanding the fact that even more efficient $T$ cell priming could have been achieved by vaccination with fully and stably matured DCs.

Prostate cancer is one of the few and unique tumor systems for which a highly specific biomarker, namely PSA, is available for monitoring disease progression. Although its use in predicting survival in a metastatic hormone refractory setting is controversial due to considerable tumor heterogeneity, its use in predicting disease progression in a minimal residual disease setting in which immunotherapy is ultimately more likely to be effective is rather well accepted. In this trial, a modest antitumor effect was suggested in six of seven evaluable patients by decreased serum PSA velocities upon initiation of vaccine therapy. We further found that vaccination is associated with a complete clearance of circulating tumor cells in patients with detectable levels of hematogenous micrometastases prior to therapy. Molecular clearance of tumor cells as a surrogate endpoint for therapeutic benefit has been reported previously (15). We have made similar observations on declining numbers of circulating prostate tumor cell upon initiation of systemic chemotherapy or androgen ablation (unpublished observations). Although the prognostic relevance of circulating tumor cell clearance is still unknown, this analysis may be used as a sensitive assay to detect clinical impact by investigational therapeutics at a molecular level. It is not inconceivable that not all prostate cancer patients, particularly those with minimal tumor burdens, will have detectable levels of circulating tumor cells before therapy, thus allowing this form of monitoring. As a potential solution to this problem, other studies suggest that occult micrometastases in bone marrow may allow a similar analysis even in patients with early disease stages (25). In summary, active immunotherapy with PSA RNA-transfected DCs represents a novel approach for prostate cancer immunotherapy. Our data provide evidence on safety and in vivo bioactivity of RNA-loaded DCs in patients with metastatic prostate cancer, although the proof for clinical benefit remains to be established in future clinical trials. This trial establishes the foundation for further optimization and refinements of the RNA-loaded DC strategy with the ultimate goal of developing therapeutic vaccines for cancer patients.

\section{Acknowledgments}

This work was supported in part by NIH grant KO8-CA-9322, the CaP CURE Foundation, and by the National Center for Research Resources, NIH grant MO1-RR-30, General Clinical Research Centers Program, NIH.

1. Small, E.J. 1998. Prostate cancer, incidence, management and outcomes. Drugs Aging. 13:71-81.

2. Hsu, F.J., et al. 1996. Vaccination of patients with B-cell lymphoma using autologous antigen-pulsed dendritic cells. Nat. Med. 2:52-58.

3. Nestle, F.O., et al. 1998. Vaccination of melanoma patients with peptideor tumor lysate-pulsed dendritic cells. Nat. Med. 4:328-332.

4. Small, E.J., et al. 2000. Immunotherapy of hormone-refractory prostate cancer with antigen-loaded dendritic cells. J. Clin. Oncol. 18:3894-3903.

5. Banchereau, J., and Steinman, R.M. 1998. Dendritic cells and the control of immunity. Nature. 392:245-252.

6. Gilboa, E., Nair, S.K., and Lyerly, H.K. 1998. Immunotherapy of cancer with dendritic-cell-based vaccines. Cancer Immunol. Immunother. 46:82-87.

7. Ashley, D.M., et al. 1997. Bone marrow-generated dendritic cells pulsed with tumor extracts or tumor RNA induce antitumor immunity against central nervous system tumors. J. Exp. Med. 186:1177-1182.

8. Boczkowski, D., Nair, S.K., Snyder, D., and Gilboa, E. 1996. Dendritic cells pulsed with RNA are potent antigen-presenting cells in vitro and in vivo. J. Exp. Med. 184:465-472.

9. Nair, S.K., et al. 1998. Induction of primary CEA-specific cytotoxic T lymphocytes in vitro using human dendritic cells transfected with RNA. Nat. Biotechnol. 16:364-369.

10. Heiser, A., et al. 2000. Human dendritic cells transfected with RNA encoding PSA stimulate prostate specific CTL responses in vitro. J. Immunol. 164:5508-5514.

11. Bryant, J., Day, R., Whiteside, T.L., and Herberman, R.B. 1992. Calculation of lytic units for the expression of cell-mediated cytotoxicity. J. Immunol. Methods. 146:91-103.

12. Kammula, U.S., Marincola, F.M., and Rosenberg, S.A. 2000. Real-time quantitative polymerase chain reaction assessment of immune reactivity in melanoma patients after tumor peptide vaccination. J. Natl. Cancer Inst. 92:1336-1344.

13. Slovin, S.F., and Scher, H.I. 1999. Peptide and carbohydrate vaccines in relapsed prostate cancer: immunogenicity of synthetic vaccines in man. Semin. Oncol. 26:448-454.

14. Small, E.J., et al. 2000. Prospective trial of the herbal supplement PC-SPES in patients with progressive prostate cancer. J. Clin. Oncol. 18:3595-3603.

15. Bendandi, M., et al. 1999. Complete molecular remissions induced by patient-specific vaccination plus granulocyte-monocyte colony-stimulating factor against lymphoma. Nat. Med. 5:1171-1177.

16. Balzar, M., Winter, M.J., de Boer, C.J., and Litvinov, S.V. The biology of the 17-1A antigen (Ep-CAM). J. Mol. Med. 77:699-712.

17. Heiser, A., Dahm, P., Maurice, M., Yancey, D., and Vieweg, J. 2001. Human dendritic cells pulsed with renal tumor RNA stimulate tumor specific CTL in vitro without inducing autoimmune toxicity. Cancer Res. 61:411-419.

18. Heiser, A, et al. Induction of polyclonal prostate cancer specific cytotoxic T lymphocytes using dendritic cells transfected with amplified tumor RNA. J. Immunol. 166:2953-2960.

19. Sanda, M.G., et al. 1999. Recombinant vaccinia-PSA (PROSTVAC) can induce a prostate-specific immune response in androgen-modulated human prostate cancer. Urology. 53:260-266.

20. Meidenbauer, N., Harris, D.T., Spitler, L.E., and Whiteside, T.L. 2000. Generation of PSA-reactive effector cells after vaccination with a PSAbased vaccine in patients with prostate cancer. Prostate. 43:88-100.

21. Murphy, G., Tjoa, B., Ragde, H., Kenny, G., and Boynton, A. 1996. Phase I clinical trial: T-cell therapy for prostate cancer using dendritic cells pulsed with HLA-201-specific peptides from prostate-specific membrane antigen. Prostate. 29:371-380.

22. Small, E. J., et al. 2000. Immunotherapy of hormone-refractory prostate cancer with antigen-loaded dendritic cells. J. Clin. Oncol. 18:3894-3903.

23. Dhodapkar, M.V., Steinman, R.M., Krasovsky, J., Munz, C., and Bhardwaj, N. 2001. Antigen-specific inhibition of effector T cell function in humans after injection of immature dendritic cells. J. Exp. Med. 193:233-238.

24. Weissman, D., et al. 2000. HIV gag mRNA transfection of dendritic cells (DC) delivers encoded antigen to MHC class I and II molecules, causes DC maturation, and induces a potent human in vitro primary immune response. J. Immunol. 165:4710-4717.

25. Braun, S., and Pantel, K. 1999. Micrometastatic bone marrow involvement: detection and prognostic significance. Med. Oncol. 16:154-165. 